\title{
Vertebroplastia percutánea guiada por tomografía para el manejo del dolor en pacientes con fractura del cuerpo vertebral
}

\section{CT-guided Vertebroplasty for Painful Vertebral Fractures}
S. Kozima ${ }^{1,2}$
A. de Salazar ${ }^{1}$
G. Espil ${ }^{2}$
C. Siffredi ${ }^{2}$
L. Ferrari ${ }^{1}$
C. Mora ${ }^{1}$
J. Garralda ${ }^{2}$
J. H. Pinzón ${ }^{1}$
R. Cobeñas ${ }^{1}$
M. Aguilar ${ }^{1}$
N. Larrañaga ${ }^{2}$
A. Salamida ${ }^{1}$

${ }^{1}$ Departamento de Imágenes, Centro de Educación Médica e
Investigaciones Clínicas (CEMIC), Ciudad Autónoma de Buenos Aires,
Argentina
2 Servicio de Diagnóstico por Imágenes, Hospital General de Agudos
Dr. Cosme Argerich, Ciudad Autónoma de Buenos Aires, Argentina

Address for correspondence Jairo Hernández Pinzón, MSc, Centro de Educación Medica e Investigaciones Clínicas, Hospital Universitario Sede Saavedra, Departamento de imágenes. Galván 4102, Capital Federal, Argentina (e-mail: jahernandezpinzon@gmail.com).

Rev Argent Radiol 2018;82:2-12.

\section{Resumen}

\section{Palabras-Clave \\ - vertebroplastia \\ - tomografía computada multidetector \\ - fractura vertebral}

Objetivo Describir la técnica y resultados en cuanto a la mejoría del dolor y complicaciones al realizar este procedimiento mediante guía por tomografía computada.

Materiales y Métodos Estudio observacional descriptivo de una serie de 108 pacientes a quienes se les realizó vertebroplastia percutánea guiada por tomografía computada realizadas en dos hospitales universitarios, entre mayo 2007 y mayo 2017. Todos los procedimientos se realizaron de forma ambulatoria con anestesia local y se valoró el dolor mediante la escala visual análoga.

Resultados Se realizaron 125 vertebroplastias, en el $87,9 \%$ de los pacientes $(n=95)$ se realizó el procedimiento en un cuerpo vertebral, en el $8,3 \%(n=9)$ y $3,7 \%(n=4)$ de los pacientes se cementaron 2 y 3 vertebras respectivamente. El rango de dolor según la escala visual análoga (EVA) previo al tratamiento varió entre 5 y 10, donde un 94\% $(n=102)$ de los pacientes manifestaban una intensidad 10/10. En el postratamiento el rango de dolor varió entre 0 a 7 donde el $98 \%$ de la población reportó un valor menor o igual a 3. Se presentaron 3 complicaciones: tromboembolismo pulmonar por metilmetacrilato, extravasación al plexo de Batson y extravasación al espacio interdiscal, cada una en tres pacientes diferentes.

Conclusión La vertebroplastia percutánea guiada por TC ofrece una indiscutible mejora inmediata del dolor en pacientes con fractura de uno o más cuerpos vertebrales, con una baja tasa de complicaciones. received July 7, 2017 accepted December 23, 2017 published online March 23, 2018
DOI https://doi.org/ 10.1055/s-0038-1637029. ISSN 1852-9992.
Copyright (c) 2019, Sociedad Argentina de Radiología. Publicado por Thieme Revinter Publicações Ltda., Rio de Janeiro, Brazil. Todos los derechos reservados.

\section{License terms}

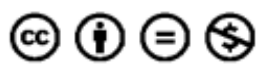




\begin{abstract}
Keywords

- vertebroplasty

- multidetector computed tomography

- spinal fracture
\end{abstract}

Objetive Describe the technique, results in terms of pain improvement and complications to perform this procedure by computed tomography.

Materials and Methods A descriptive observational study of a 108 cases series of percutaneous vertebroplasty guided by computed tomography performed in two university hospitals between May 2007 and May 2017. All procedures were performed with local anesthesia on an outpatient basis, pain was assessed by means of the Visual analogue scale (VAS).

Results A total of 125vertebroplasties were performed. In $87.9 \%(n=95)$ of the patients, the procedure was performed in one vertebral body, in $8.3 \%(n=9)$ and $3.7 \%$ $(n=4)$ of the patientshad two or three vertebrae cemented respectively. The range of pain according to VAS prior to treatment varied between 5 and 10 , where $94 \%$ ( $n=102$ ) of the patients manifested a 10/10 intensity; after treatment, the range of pain varied between 0 to 7 where $98 \%$ of the population reported a value less than or equal to 3. Three complications were reported, one pulmonary thromboembolism due to methylmethacrylate, one extravasation in to the Batson plexus and one extravasation of cement to the interdiscal space.

Conclusion CT-guided percutaneous vertebroplasty offers an undeniable immediate improvement of pain in patients with fracture of one or more vertebral bodies, with a low rate of complications.

\section{Introducción}

La vertebroplastia percutánea (VTP) consiste en la inyección percutánea de cemento óseo (usualmente metilmetacrilato). Es una técnica que se comenzó a utilizar a mediados de los años 80 siendo inicialmente descripta en el tratamiento del hemangioma vertebral sintomático. ${ }^{1,2}$ Desde entonces, las indicaciones de ese método se extendieron para el tratamiento de las fracturas compresivas del cuerpo vertebral relacionadas a trauma, osteoporosis, metástasis y mieloma múltiple. ${ }^{3-6}$

Se considera que la técnica permite aliviar el dolor asociado a las fracturas por compresión mediante la ruptura de los receptores de dolor y reducir las fuerzas dinámicas de presión que se ejercen sobre los cuerpos vertebrales dañados mediante la inyección intraósea del metil-metacrilato, ${ }^{7}$ en ausencia de síntomas directos de compresión, destrucción de la pared vertebral y la presencia de compromiso epidural que pueden ser contraindicaciones relativas del procedimiento. ${ }^{8}$

El objetivo de este trabajo es describir la técnica y resultados en cuanto a la mejoría del dolor, al igual que las posibles complicaciones al realizar este procedimiento mediante guía por tomografía computada (TC).

\section{Materiales y Métodos}

Este trabajo fue aprobado por el comité de ética de nuestra institución. Es un estudio observacional descriptivo de una serie de 108 casos a quienes se les realizó como parte de su manejo terapéutico estándar del dolor VTP bajo control por TC en el período comprendido entre mayo del 2007 y mayo del
2017, en dos hospitales universitarios. Todos los procedimientos fueron realizados por el mismo radiólogo intervencionista, con la ayuda de médicos especialistas y residentes en diagnóstico por imágenes.

Los criterios para realizar el procedimiento fueron: fractura de uno o más cuerpos vertebrales de hasta 12 meses de evolución, con o sin daño de la cortical ósea o dispersión de los fragmentos. Los criterios para no realizar el procedimiento fueron: ausencia de muro posterior en el cuerpo vertebral afectado, acuñamiento vertebral, ausencia de médula ósea en el cuerpo vertebral remanente y pacientes con coagulopatía que no pudiera corregirse.

Se utilizaron equipos de TC: Philips Gemini (Eindhoven, Países Bajos) de 64 detectores, Philips MX (Cleveland, Ohio, USA) de 16 detectores y Toshiba Aquilion (Tokio, Japón) de 16 detectores. Los parámetros de adquisición de las imágenes fueron: en primera adquisición $1 \mathrm{~mm}$ cada $0.5 \mathrm{~mm}$ de espesor, FOV 340-450, matriz de 512, Kv 120 mA 100. Una vez seleccionada la vértebra fracturada, tomadas las medidas y marcado el paciente, se realizaron las subsiguientes series de $3 \mathrm{~mm}$ cada $3 \mathrm{~mm}$, aproximadamente 20 cortes con el mismo FOV por serie. Se trabajó con un filtro estándar; realizando la reconstrucción en cortes axiales, sagitales y coronales para guiar el sitio de la punción, la dirección y el punto hasta donde avanzar la aguja.

\section{Técnica Utilizada}

Los pasos utilizados para la realización de la VTP guiada por TC se ilustran en la (-Fig. 1). El procedimiento fue en todos los casos, realizado utilizando anestesia local, con monitoreo constante de signos vitales y en forma ambulatoria. Una vez 

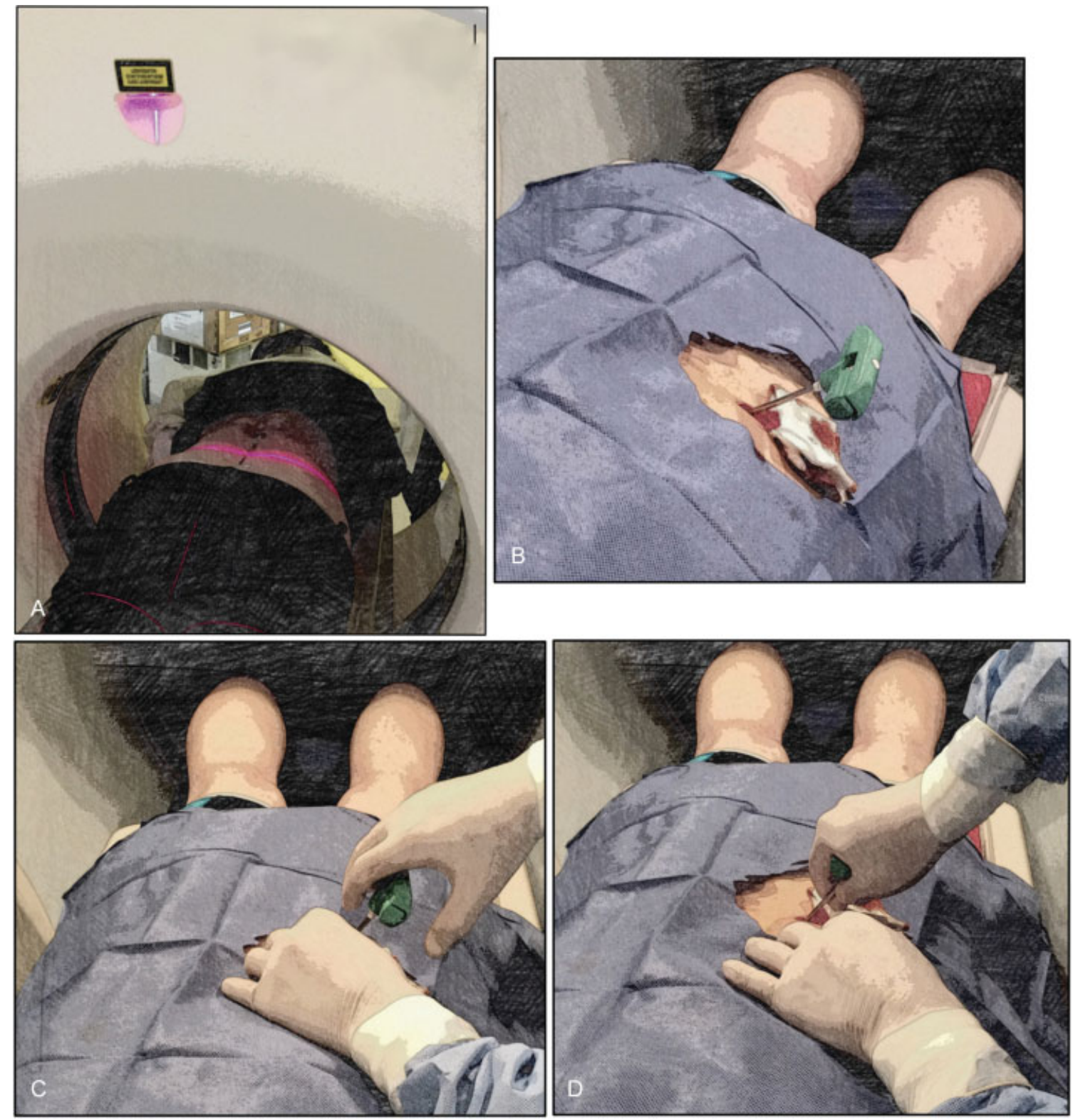

Fig. 1 (A) Siguiendo la guía de la luz láser del tomógrafo marcamos la piel en el sitio de punción, de acuerdo al espesor residual del cuerpo vertebral a tratar. Se utilizó una aguja de 8 a 11 G. (B) Previa desinfección de partes blandas y anestesia local con lidocaína, utilizando una jeringa de 10 cc, realizamos un habón principalmente a nivel dérmico - subdérmico (3 cc); en el trayecto del tejido celular subcutáneo (3 cc) y al llegar al periostio el resto de los $10 \mathrm{cc}$, luego de una pequeña incisión con un bisturí en la piel, se posiciona la aguja de punción ósea en el trayecto planificado. (Cy D) Posteriormente con movimientos de giro-compresión hasta traspasar el periostio se deja fija la aguja sin penetrar en lo posible a la médula ósea. (E) Se realiza un primer control con el fin de comprobar la posición de la aguja y corregir si es necesario la dirección hacia el centro del cuerpo vertebral. En el caso de no poder acceder al centro del mismo realizamos la punción bilateral (lo cual sucedió en 1 caso). (F) Una vez posicionada la aguja, preparamos el cemento acrílico; para la mezcla se utilizó el mismo envase plástico de la ampolla en la que vertemos el polvo de metil-metacrilato con bario (subiton RO o vertebro) y el solvente para preparar el cemento, mezclamos hasta alcanzar la consistencia de una pasta dental. $(\mathrm{G} \mathrm{y} \mathrm{H}$ ) Retiramos el mandril de la aguja e inyectamos con una jeringa de 5 cc que se carga con 3,5 cc del material preparado e inyectamos manualmente. Habitualmente esa maniobra manual nos permite inyectar hasta unos $2 \mathrm{cc}(\mathrm{I})$ en bolo (dependiendo del grado de acuñamiento y de fracturas del cuerpo vertebral), (J) con el mandril empujamos el material que queda en la aguja y continuamos inyectando más material hasta completar 3 cc o hasta que la resistencia al empuje del mismo impida progresar el mandril (con el cemento aún no habiendo alcanzado su consistencia sólida). (K) Retiramos la aguja y se realiza control tomográfico.

finalizado el procedimiento, se colocó al paciente en decúbito dorsal durante dos horas aproximadamente para el manejo de la hemostasia y del dolor, posiblemente provocado debido a la punción o a la reacción inflamatoria generada secundaria al calor por la solidificación del metacrilato.
Para el tratamiento del dolor local se administraron antiinflamatorios no esteroideos hasta dos días siguientes; se evaluó el dolor mediante la escala visual análoga (EVA) con un rango de 0 (ningún dolor) a 10 (máximo dolor) pasadas dos horas del procedimiento con el paciente en bipedestación. 

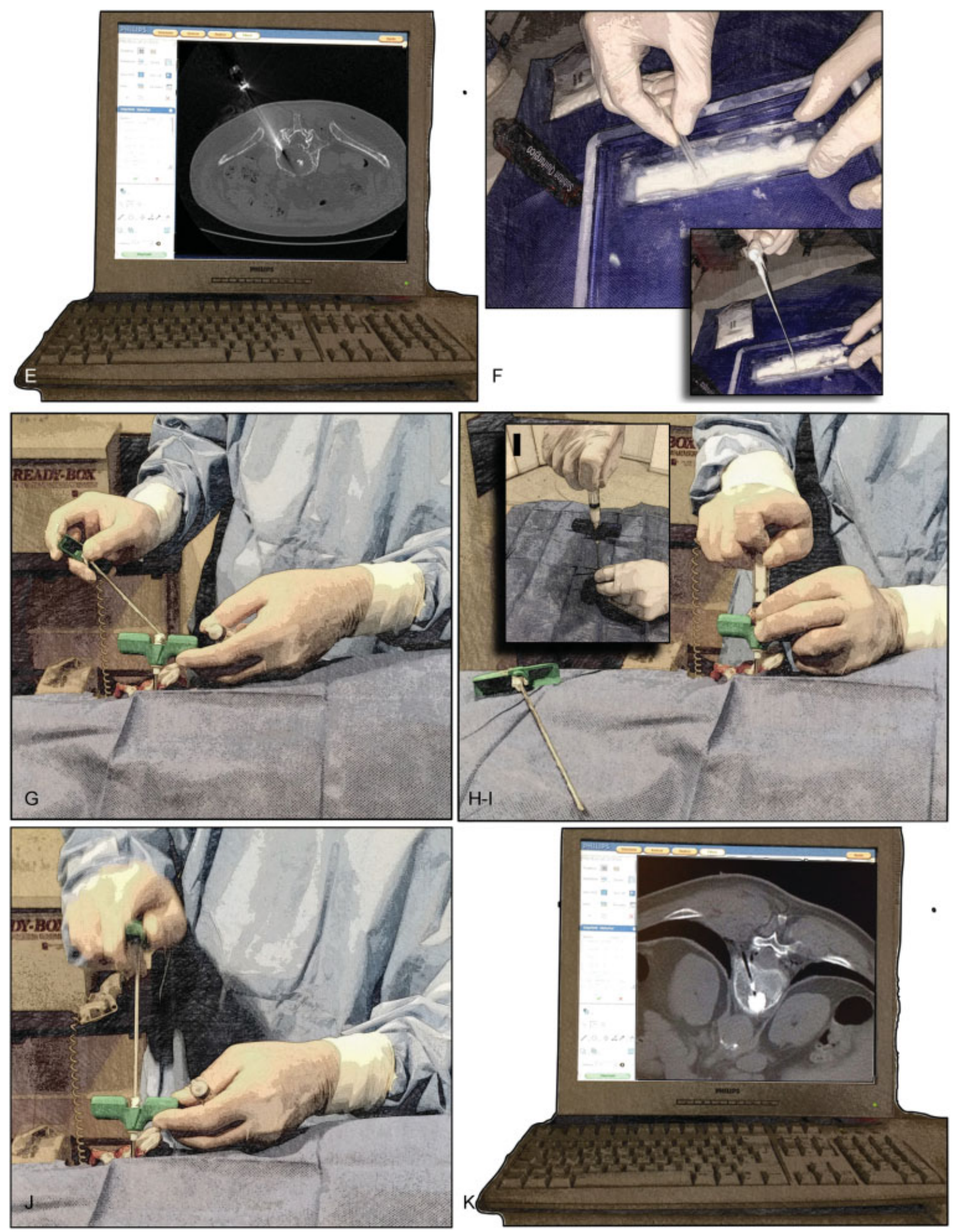

Fig. 1 (Continued)

Se recolectaron los datos de forma prospectiva en una hoja de cálculo; se procesaron los datos mediante el programa estadístico Stata 14 (StataCorp LLC, College Station Texas), reportando los mismos mediante estadísticas descriptivas.

\section{Resultados}

Se realizaron 125 VTP en 108 pacientes (19 hombres y 89 mujeres) con una edad media de 71 años $+/-10,65$ y un rango de entre 25 y 101 años (-Fig. 2). 

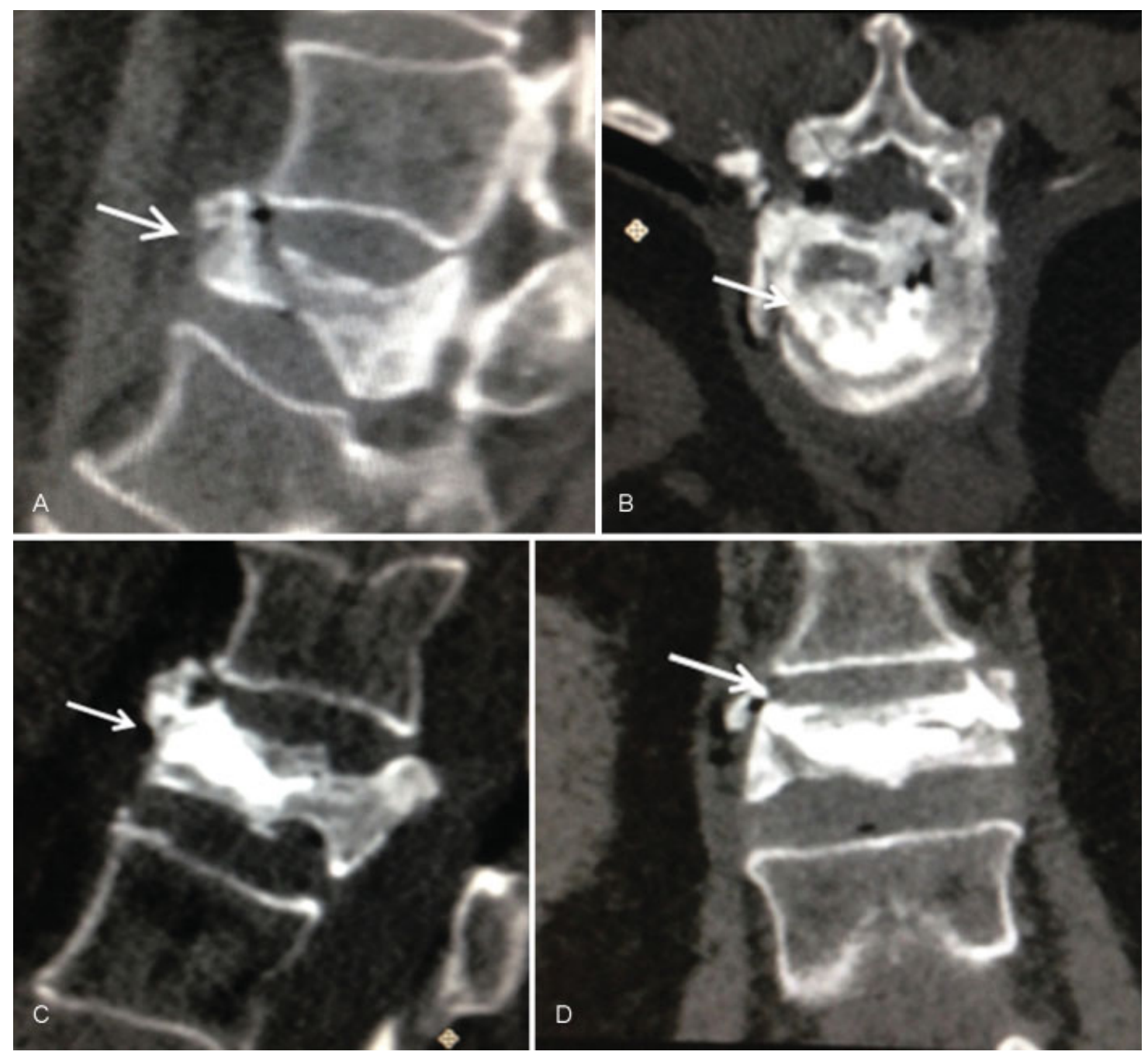

Fig. 2 Ejemplo de procedimiento. Paciente que presentó fractura de acuñamiento de cuerpo vertebral L1. Se realizó tomografía computada (TC) de columna lumbar (A) plano sagital, se observó acuñamiento vertebral previo al tratamiento. El plano axial (B), el plano sagital (C) y el plano coronal (D), muestran la ubicación del cemento en el cuerpo vertebral al finalizar el procedimiento (flechas).

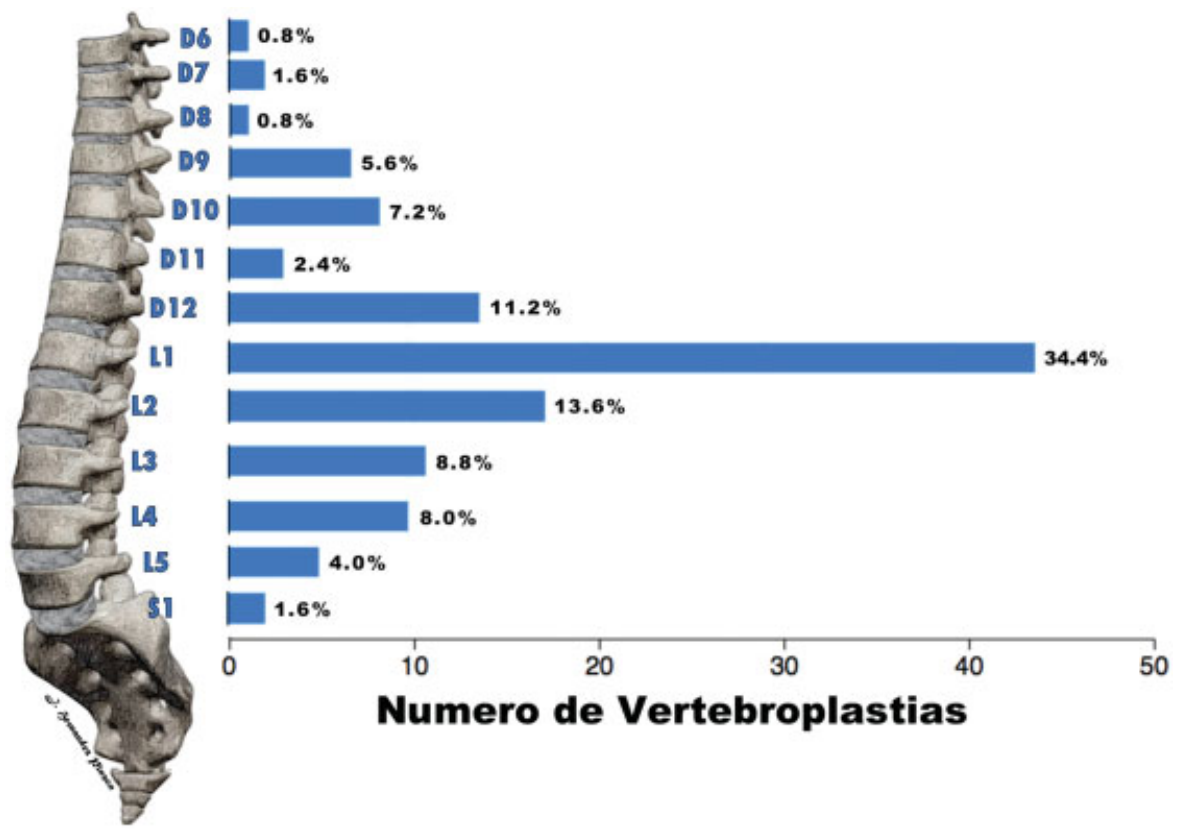

Fig. 3 Porcentaje y número de vertebroplastias percutáneas (VTP) de acuerdo al cuerpo vertebral. 
Tabla 1 Porcentaje y frecuencia de las principales causas de vertebroplastia percutánea (VTP)

\begin{tabular}{|l|l|l|}
\hline Etiología & Frecuencia & $\%$ \\
\hline F. Traumática & 103 & 95 \\
\hline F. Osteoporótica & 1 & 1 \\
\hline Metástasis líticas & 2 & 2 \\
\hline Mieloma & 1 & 1 \\
\hline Enf. de Still & 1 & 1 \\
\hline Total & $\mathbf{1 0 8}$ & $\mathbf{1 0 0}$ \\
\hline
\end{tabular}

En el $87,9 \%(n=95)$ de los pacientes se realizó el procedimiento en un cuerpo vertebral, en el $8,3 \%(n=9)$ y en el $3,7 \%(n=4)$ de los pacientes se cementaron 2 y 3 vértebras respectivamente. El cuerpo vertebral en el que más VTP se realizaron fue L1, seguido de L2 y D12 (-Fig. 3).

Las principales indicaciones del procedimiento fueron fracturas traumáticas y osteoporóticas, metástasis líticas, mielomas y enfermedad de Still (-Tabla 1). El rango de dolor según la EVA previo al tratamiento varió entre 5 y 10 , donde $94 \%$ ( $n=102)$ de los pacientes manifestaban una intensidad 10/10; postratamiento el rango de dolor varió

Tabla 2 Escala visual análoga (EVA) pre y post VTP

\begin{tabular}{|c|c|c|c|c|}
\hline EVA & Frecuencia pre VTP & Porcentaje pre VTP & Frecuencia post VTP & Porcentaje post VTP \\
\hline 0 & 0 & 0 & 63 & 58 \\
\hline 1 & 0 & 0 & 25 & 23 \\
\hline 2 & 0 & 0 & 13 & 12 \\
\hline 3 & 0 & 0 & 5 & 5 \\
\hline 4 & 0 & 0 & 0 & 0 \\
\hline 5 & 1 & 1 & 1 & 1 \\
\hline 7 & 2 & 2 & 1 & 1 \\
\hline 8 & 2 & 2 & 0 & 0 \\
\hline 9 & 1 & 1 & 0 & 0 \\
\hline 10 & 102 & 94 & 0 & 0 \\
\hline Total & 108 & 100 & 108 & 100 \\
\hline
\end{tabular}
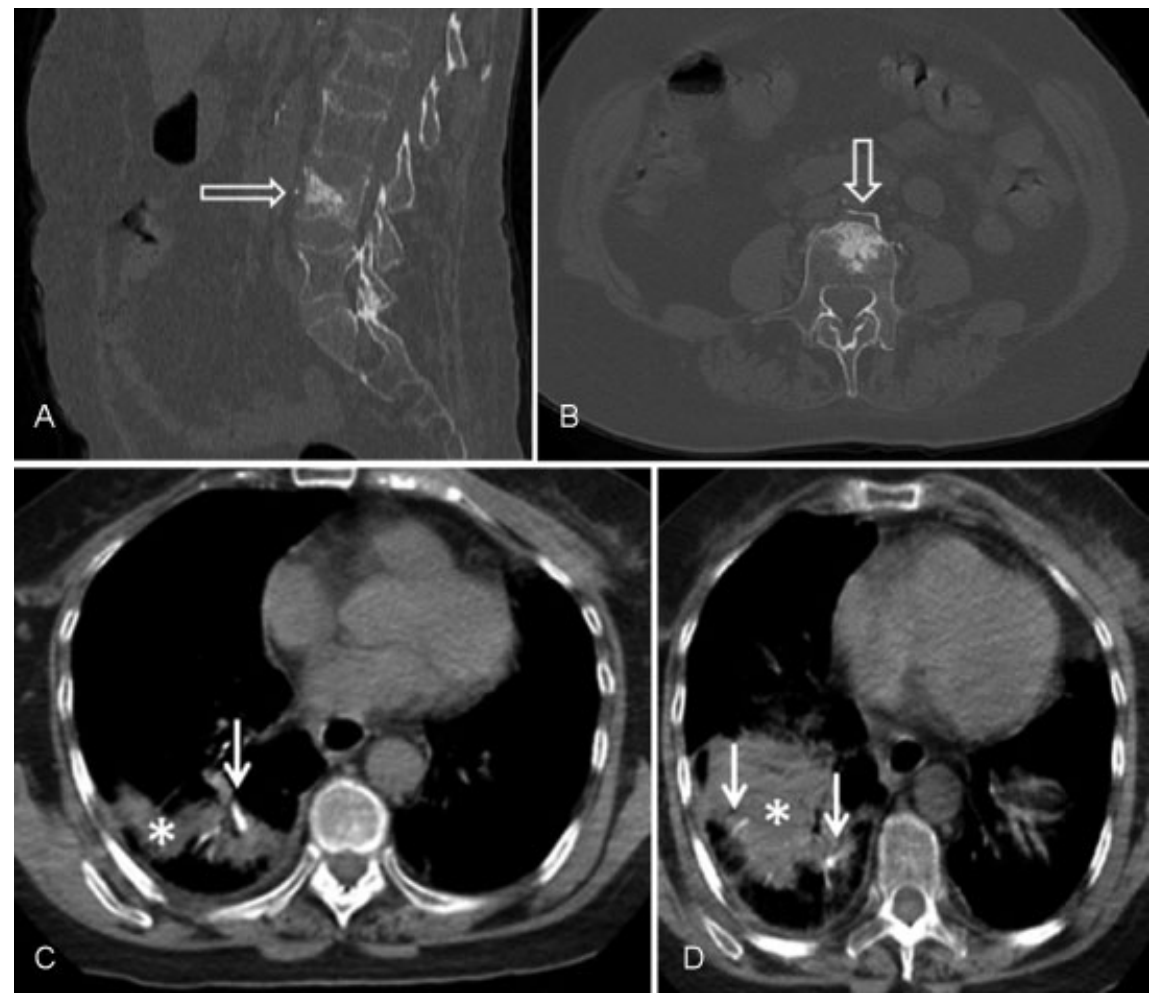

Fig. 4 Paciente de sexo femenino que presentó fractura en cuerpo vertebral L4. Se le realizó vertebroplastia y tuvo como complicación embolia hacia el plexo venoso vertebral anterior externo (flechas transparentes), (A) TC de columna lumbar en plano sagital y (B) TC de columna lumbar en plano axial. (C) y (D) TC de tórax sin contraste, plano axial; en lóbulo inferior derecho, se observan imágenes lineales hiperdensas en relación a ramas distales de la arteria pulmonar, correspondientes a émbolos de cemento a dicho nivel (flechas blancas), asociadas a infarto pulmonar (asterisco). 
entre 0 a 7 donde el $98 \%$ de la población reportó un valor menor o igual a 3 (-Tabla 2).

La inyección de no más de 3cc a la consistencia mencionada no presentó volcado hacia el canal medular en ningún caso; las principales complicaciones fueron tromboembolismo pulmonar (TEP) en un paciente (-Fig. 4), extravasación del material de contraste al plexo venoso de Batson en un paciente (-Fig. 5) y al espacio intervertebral en otro paciente (-Fig. 6). En el primer caso la paciente presentó dolor lumbar cercano al sitio de punción, aunque no exactamente en el nivel vertebral cementado, se le suministraron analgésicos no esteroideos con lo que mejoró su síntoma y fue dada de alta. Al día siguiente experimentó dolor pleurítico, por lo que se le realizó una TC de tórax en la que se observó material espontáneamente denso ocupando la luz de una arteria pulmonar segmentaria; la paciente fue internada por 72 horas, recibió anticoagulación, desaparecieron los síntomas y fue dada de alta sin complicaciones posteriores.

Los otros dos casos fueron asintomáticos, dados de alta en las 2 horas posteriores al procedimiento y fueron controlados a las 24 y 36 horas, sin complicaciones posteriores. En tres casos se requirió acceso bilateral para la VTP (-Fig. 7). En un paciente no pudo ser realizado el procedimiento debido al grado de acuñamiento vertebral ( - Fig. 8), por lo cual no fue incluido en el análisis estadístico.

\section{Discusión}

La VTP ha surgido como una modalidad ampliamente aceptada para el manejo del dolor refractario por fracturas del cuerpo vertebral. ${ }^{9}$ En nuestras instituciones esa técnica se ha puesto en práctica desde el año 2007 como una valiosa opción terapéutica, con un significativo beneficio para el paciente en lo que respecta al manejo
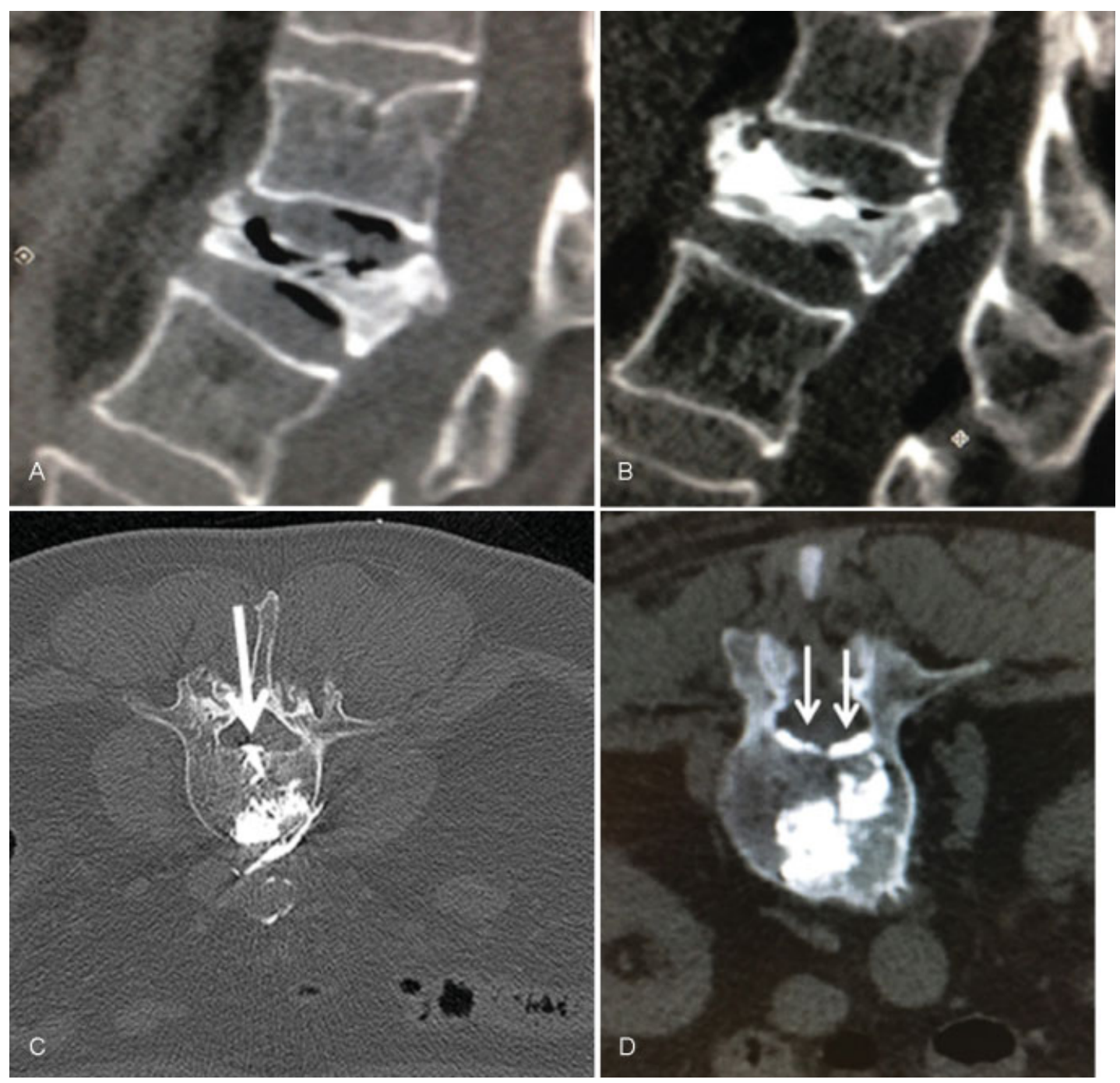

Fig. 5 Paciente con fractura aplastamiento de cuerpo vertebral L1. (A) TC de columna lumbar, en plano sagital, donde se observa la fractura antes del procedimiento. (B) TC de columna lumbar, en plano sagital, se visualiza el material de vertebroplastia en control durante el procedimiento. (C) y (D) TC de columna lumbar, en plano axial, que muestra el paso del cemento al plexo de Batson (flechas). 
del dolor, disminución del consumo de analgésicos y recuperación de la movilidad.

La guía por TC es útil para el control de la pared vertebral posterior, que resulta difícil cuando únicamente se utiliza radioscopia lateral como único plano de visualización, sin embargo en cuanto a la monitorización de la fuga de cemento craneocaudal, la guía por radioscopia tiene un buen desempeño. ${ }^{10}$ Eso se suma al hecho de que el control en tiempo real ha hecho que ese método de imágenes sea el preferido para guiar las VTP por la mayoría de radiólogos intervencionistas.

En nuestro caso, los motivos que nos hicieron optar por la guía mediante TC fueron la posibilidad de realizar reconstrucciones en los 3 planos de la vértebra afectada y sobre las mismas programar el sitio y la dirección de la punción en el plano axial, permitiendo así la posibilidad de llevar a un sitio preciso la inyección del metacrilato y de esa forma evitar la punción vertebral bilateral como lo realizan algunos autores. $^{11}$

Esa opción de reconstrucción vertebral en los tres planos es una excelente herramienta para el posicionamiento de la aguja con el fin de inyectar el cemento en el centro del cuerpo vertebral, y para ese fin llega a ser incluso superior que la guía por radioscopia, al igual que para la detección de cemento extra óseo como lo reportan Pitton y col. ${ }^{10}$ y Schmidt y col. ${ }^{12}$ respectivamente.

En nuestro caso, hemos comprobado con el control por TC un adecuado posicionamiento de la cánula con el fin de inyectar el cemento y además un buen control en la detección de fuga de cemento al plexo venoso de Batson como lo reportan otros autores. ${ }^{10}$ En este estudio, la mayoría de pacientes a quienes les realizamos VTP padecían de fracturas traumáticas y eran del sexo femenino. A pesar de la clara mejoría del dolor posterior al procedimiento es importante mencionar que existen muy pocos estudios aleatorios y ciegos que tengan una cohorte con tratamientos de control o placebo a fin de comprobar la verdadera eficacia y efectividad de ese procedimiento. Los dos primeros ensayos clínicos de ese tipo fueron publicados en agosto de $2009^{13,14}$ y dejaron la sensación de que ese procedimiento no era mejor que el placebo; sin embargo, en ambos, el placebo implicó la inyección de un analgésico de acción corta en el periostio óseo, por lo que consideramos que en esos estudios el placebo fue en realidad un tratamiento activo.

Contrario a lo que reportaron los estudios antes mencionados, una investigación prospectiva, no cegada, (The VERTOS Study), ${ }^{15}$ demostró que la VTP fue mejor
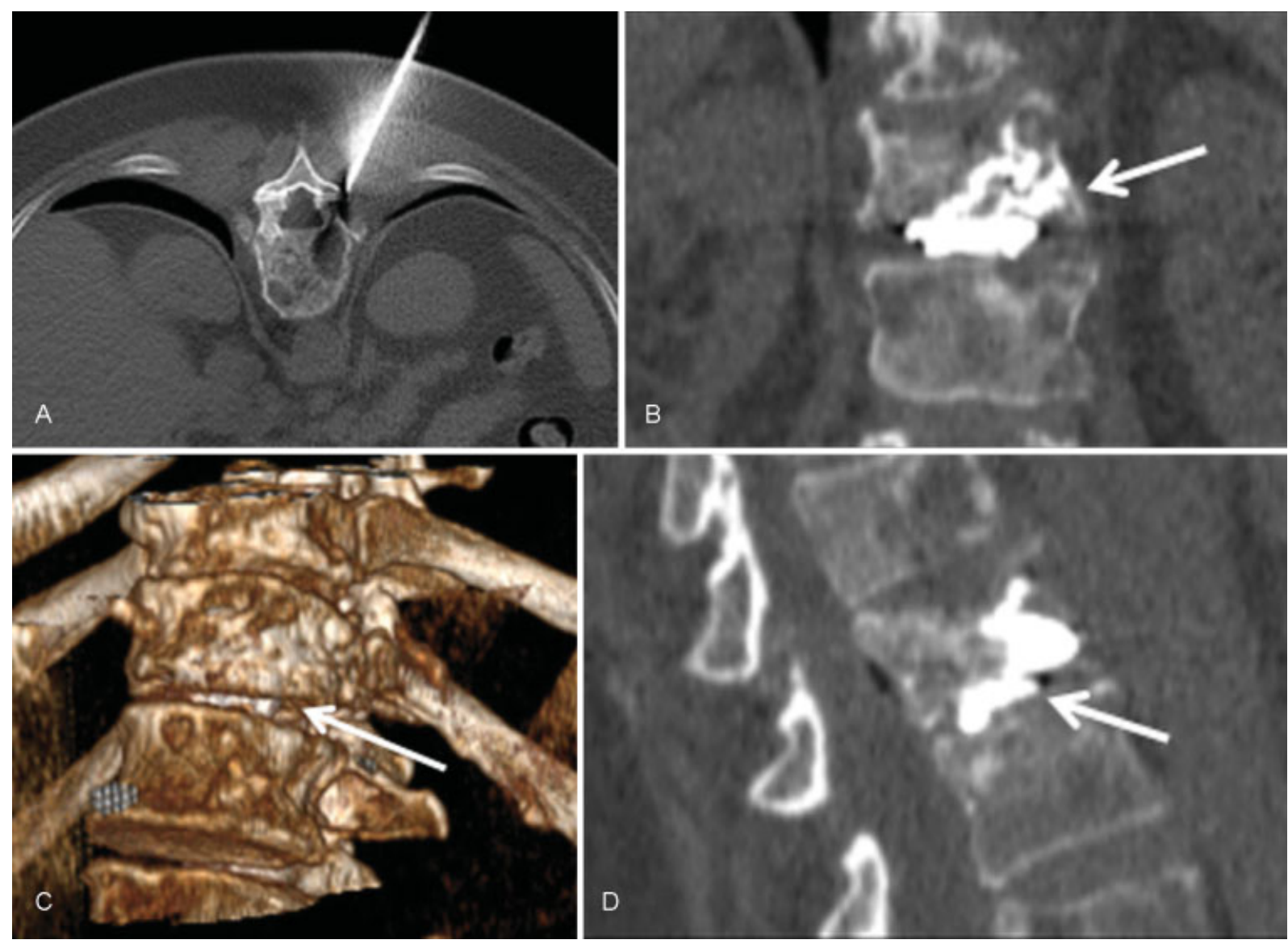

Fig. 6 Paciente con fractura de cuerpo vertebral T12. TC de columna lumbar: (A) plano axial, donde se observa el posicionamiento de la aguja ósea a nivel del pedículo izquierdo. (B) plano coronal, (C) reconstrucción en 3D y (D) plano sagital, donde se observa paso del cemento de vertebroplastia hacia el espacio intervertebral T12-L1 (flecha blanca). 


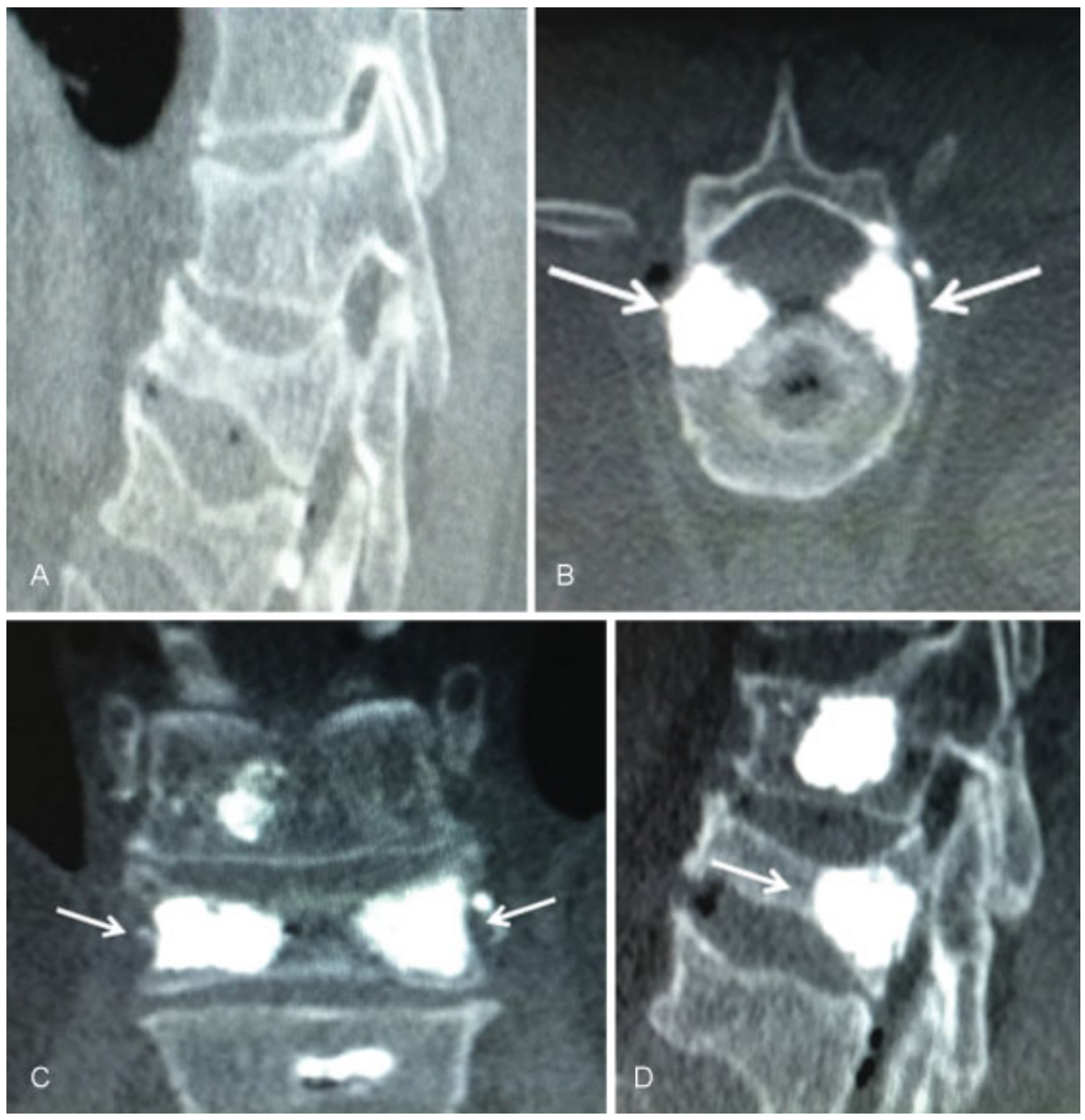

Fig. 7 Paciente que sufrió fractura en los cuerpos vertebrales T12 y L1, que requirió acceso bilateral para la vertebroplastia en L1. TC de columna lumbar que muestra (A) en plano sagital, la fractura aplastamiento previa al tratamiento y en plano axial (B), en plano coronal (C) y en plano sagital (D), muestran el material denso posterior a la inyección del polimetil-metacrilato (PMMA) (flechas).

para aliviar el dolor en comparación con el tratamiento médico óptimo al día y a las 2 semanas, resultados que están más acordes a nuestra experiencia con ese procedimiento.

Es importante mencionar que la VTP no es un procedimiento exento de riesgos, existen varios reportes de lesiones en tejidos blandos, compresión de raíces nerviosas y embolia pulmonar e insuficiencia cardiorrespiratoria por la propagación de cemento a través de las venas perivertebrales y cava inferior hacia la circulación pulmonar, hecho que se ha reportado en otras publicaciones, ${ }^{16}$ estimándose retrospectivamente entre un $4,6 \%$ y un $8,1 \%$ de embolismos pulmonares por cemento. ${ }^{17}$

Es sabido que una baja viscosidad del cemento quirúrgico se asocia a su fuga a los tejidos adyacentes ${ }^{18}$; creemos que una viscosidad lo suficientemente alta sin que ésta produzca resistencia a la inyección del material, reduce las fugas y por esa razón utilizamos en nuestros pacientes una consistencia semejante a la de una pasta dental, sumado a la cantidad de $3 \mathrm{~mL}$ como máximo que aplicamos (Pittonet y col. ${ }^{10}$ usaron $4,5 \mathrm{~mL}$ en promedio), pueden ser los factores responsables de nuestra baja tasa de complicaciones.

La complicación más grave experimentada fue un caso de tromboembolismo pulmonar (TEP) por el cemento quirúrgico, número que se encuentra por debajo de la media en cuanto a lo reportado en la revision sistemática de Krueger y col. ${ }^{19}$ y bastante por debajo de los estudios que incluyeron e identificaron como que si buscaron sistemáticamente y reportaron el riesgo de su muestra estudiada (entre 3,5\% y 23\%). En nuestro caso, a pesar de no utilizar radioscopia, no tuvimos ninguna fuga de cemento al espacio intramedular, como lo han reportado otros autores $\left(1 \%\right.$ en la serie de Pittonet y col. $\left.{ }^{10}\right)$.

Resulta importante mencionar la forma en que fue realizado ese procedimiento, se valió únicamente de anestesia local evitando riesgos cardiorrespiratorios en pacientes de edad avanzada. Al mismo tiempo, permitió la opción de una comunicación constante con el paciente durante el procedimiento a fin de detectar dolor radicular, déficit neurológico o complicaciones locales.

Entre las debilidades de este trabajo podemos mencionar que no usamos como criterios de exclusión los mencionados por la Cardiovascular and Interventional Radiological Society of Europe (CIRSE) en su última guía Standard of Practice, ${ }^{20}$ la falta de seguimiento sistemática y a largo plazo de los pacientes que no nos 


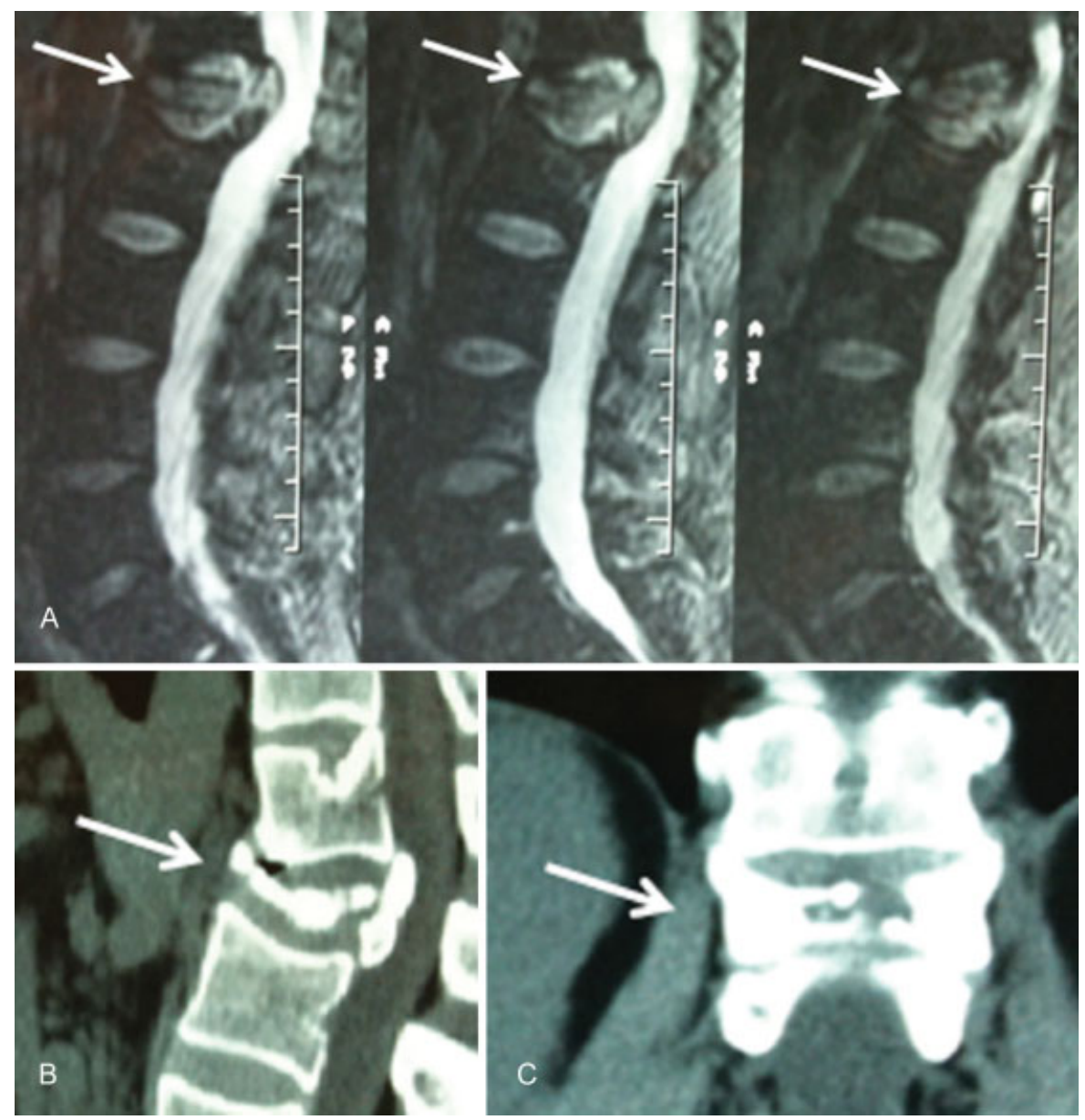

Fig. 8 Paciente con fractura aplastamiento del cuerpo vertebral L1, grado 3 en la escala de Genant, que no permite la colocación del cemento. (A) Resonancia magnética (RM) de columna lumbar, secuencia ponderada en T2 en plano sagital, (B) TC de columna lumbar en plano sagital y (C) TC de columna lumbar en plano coronal, donde se observa el cuerpo vertebral aplastado (flechas).

permitió describir con más precisión su evolución, ni los eventos adversos tardíos como fracturas en los cuerpos vertebrales adyacentes y embolismos tardíos, como lo han reportado otros autores. ${ }^{10,17}$ Así mismo, al ser este trabajo descriptivo de una serie de casos, presenta las limitaciones comunes a este tipo de diseño epidemiológico como lo son la subjetividad personal en el reporte de los resultados previos y posteriores al procedimiento, lo cual puede ser un posible sesgo de medición. También existe una falta de comparación con otros tratamientos o técnicas de VTP y finalmente debemos mencionar que al representar la experiencia de un solo radiólogo intervencionista que realizó todos los procedimientos se limita la replicación de nuestros resultados por parte de otros autores.

\section{Conclusión}

En nuestro medio, la realización de la vertebroplastia acrílica percutánea ofrece una indiscutible mejoría inmediata del dolor en pacientes con fractura de uno o más cuerpos vertebrales, siendo un procedimiento que se puede realizar guiado por TC de manera sencilla y en forma ambulatoria, con anestesia local y con una muy baja tasa de complicaciones.

\section{Responsabilidades éticas}

Protección de personas y animales. Los autores declaran que para esta investigación no se han realizado experimentos en seres humanos ni en animales.

Confidencialidad de los datos. Los autores declaran que han seguido los protocolos de su centro de trabajo sobre la publicación de datos de pacientes.

Derecho a la privacidad y consentimiento informado. Los autores declaran que en este artículo no aparecen datos de pacientes.

\section{Conflicto de Intereses}

Los autores declaran no tener ningún conflicto de interés, excepto el Dr. Kozima que declara como posible conflicto de interés ser miembro de la comisión directiva de la SAR y, los Dres. Espil y Hernández Pinzón que declaran como posible conflicto de interés ser revisores de la RAR. 


\section{Bibliografía}

1 Galibert P, Deramond H, Rosat P, Le Gars D. [Preliminary note on the treatment of vertebral angioma by percutaneous acrylic vertebroplasty]. Neurochirurgie 1987;33(02):166-168

2 Peh WCG, Gilula LA, Peck DD. Percutaneous vertebroplasty for severe osteoporotic vertebral body compression fractures. Radiology 2002;223(01):121-126

3 Barragán-Campos HM, Vallée J-N, Lo D, et al. Percutaneous vertebroplasty for spinal metastases: complications. Radiology 2006;238(01):354-362

4 Calmels V, Vallée J-N, Rose M, Chiras J. Osteoblastic and mixed spinal metastases: evaluation of the analgesic efficacy of percutaneous vertebroplasty. AJNR Am J Neuroradiol 2007;28 (03):570-574

5 Cotten A, Dewatre F, Cortet B, et al. Percutaneous vertebroplasty for osteolytic metastases and myeloma: effects of the percentage of lesion filling and the leakage of methyl methacrylate at clinical follow-up. Radiology 1996;200(02):525-530

6 Mont'Alverne F, Vallée JN, Cormier E, et al. Percutaneous vertebroplasty for metastatic involvement of the axis. AJNR Am J Neuroradiol 2005;26(07):1641-1645

7 Jensen ME, Evans AJ, Mathis JM, Kallmes DF, Cloft HJ, Dion JE. Percutaneous polymethylmethacrylate vertebroplasty in the treatment of osteoporotic vertebral body compression fractures: technical aspects. AJNR Am J Neuroradiol 1997;18 (10):1897-1904

8 Saliou G, Kocheida M, Lehmann P, et al. Percutaneous vertebroplasty for pain management in malignant fractures of the spine with epidural involvement. Radiology 2010;254(03): 882-890

9 Debussche-Depriester C, Deramond H, Fardellone P, et al. Percutaneous vertebroplasty with acrylic cement in the treatment of osteoporotic vertebral crush fracture syndrome. En: Proceedings of the XIV Symposium Neuroradiologicum. Berlín: Springer; 1991:149-152

10 Pitton MB, Herber S, Koch U, Oberholzer K, Drees P, Düber C. CTguided vertebroplasty: analysis of technical results, extraosseous cement leakages, and complications in 500 procedures. Eur Radiol 2008;18(11):2568-2578

11 Tan Z, Di Z, Mao X, Zhang J, Zou R, Wang Q. Percutaneous vertebroplasty guided by preoperative computed tomography measurements. Indian J Orthop 2016;50(06):622-628

12 Schmidt R, Cakir B, Mattes T, Wegener M, Puhl W, Richter M. Cement leakage during vertebroplasty: an underestimated problem? Eur Spine J 2005;14(05):466-473

13 Kallmes DF, Comstock BA, Heagerty PJ, et al. A randomized trial of vertebroplasty for osteoporotic spinal fractures. N Engl J Med 2009;361(06):569-579

14 Buchbinder R, Osborne RH, Ebeling PR, et al. A randomized trial of vertebroplasty for painful osteoporotic vertebral fractures. $\mathrm{N}$ Engl J Med 2009;361(06):557-568

15 Voormolen MHJ, Mali WPTM, Lohle PNM, et al. Percutaneous vertebroplasty compared with optimal pain medication treatment: short-term clinical outcome of patients with subacute or chronic painful osteoporotic vertebral compression fractures. The VERTOS study. AJNR Am J Neuroradiol 2007;28(03):555-560

16 Saracen A, Kotwica Z. Complications of percutaneous vertebroplasty. Medicine (Baltimore) [Internet]. Junio 2016;95 (24). Disponible en: http://www.ncbi.nlm.nih.gov/pmc/articles/ PMC4998452/. (accedido Junio 2017)

17 Layton KF, Thielen KR, Koch CA, et al. Vertebroplasty, first 1000 levels of a single center: evaluation of the outcomes and complications. AJNR Am J Neuroradiol 2007;28(04):683-689

18 Baroud G, Crookshank M, Bohner M. High-viscosity cement significantly enhances uniformity of cement filling in vertebroplasty: an experimental model and study on cement leakage. Spine 2006;31(22):2562-2568

19 Krueger A, Bliemel C, Zettl R, Ruchholtz S. Management of pulmonary cement embolism after percutaneous vertebroplasty and kyphoplasty: a systematic review of the literature. Eur Spine J 2009;18(09):1257-1265

20 Tsoumakidou G, Too CW, Koch G, et al. CIRSE Guidelines on Percutaneous Vertebral Augmentation. Cardiovasc Intervent Radiol 2017;40(03):331-342 